\title{
NGC 404: A REJUVENATED LENTICULAR GALAXY ON A MERGER-INDUCED, BLUEWARD EXCURSION INTO THE GREEN VALLEY
}

\author{
David A. Thilker ${ }^{1}$, Luciana Bianchi $^{1}$, David Schiminovich $^{2}$, Armando Gil de Paz $^{3}$, Mark Seibert ${ }^{4}$, Barry F. Madore ${ }^{4}$, \\ Ted Wyder ${ }^{5}$, R. Michael Rich ${ }^{6}$, Sukyoung Yi ${ }^{7}$, Tom Barlow ${ }^{5}$, Tim Conrow ${ }^{5}$, Karl Forster $^{5}$, Peter Friedman $^{5}$, \\ Chris Martin $^{5}$, Patrick Morrissey $^{5}$, Susan NeFF $^{8}$, and Todd Small $^{5}$ \\ ${ }^{1}$ Center for Astrophysical Sciences, The Johns Hopkins University, 3400 N. Charles Street, Baltimore, MD 21218, USA; dthilker@pha.jhu.edu \\ ${ }^{2}$ Department of Astronomy, Columbia University, 550 West 120th Street, New York, NY 10027, USA \\ ${ }^{3}$ Departamento de Astrofísica y CC. de la Atmósfera, Universidad Complutense de Madrid, Avda. de la Complutense, s/n, E-28040 Madrid, Spain \\ ${ }^{4}$ Observatories of the Carnegie Institution of Washington, 813 Santa Barbara Street, Pasadena, CA 91101, USA \\ ${ }^{5}$ California Institute of Technology, MC 405-47, 1200 East California Boulevard, Pasadena, CA 91125, USA \\ ${ }^{6}$ Department of Physics and Astronomy, UCLA, Los Angeles, CA 90095-1547, USA \\ ${ }^{7}$ Department of Astronomy, Yonsei University, Seoul 120-749, Republic of Korea \\ ${ }^{8}$ Laboratory for Astronomy and Solar Physics, NASA Goddard Space Flight Center, Greenbelt, MD 20771, USA \\ Received 2009 October 6; accepted 2010 March 24; published 2010 April 9
}

\begin{abstract}
We have discovered recent star formation in the outermost portion $\left((1-4) \times R_{25}\right)$ of the nearby lenticular (S0) galaxy NGC 404 using Galaxy Evolution Explorer UV imaging. FUV-bright sources are strongly concentrated within the galaxy's H I ring (formed by a merger event according to del Río et al.), even though the average gas density is dynamically subcritical. Archival Hubble Space Telescope imaging reveals resolved upper mainsequence stars and conclusively demonstrates that the UV light originates from recent star formation activity. We present FUV, NUV radial surface brightness profiles, and integrated magnitudes for NGC 404. Within the ring, the average star formation rate (SFR) surface density $\left(\Sigma_{\mathrm{SFR}}\right)$ is $\sim 2.2 \times 10^{-5} M_{\odot} \mathrm{yr}^{-1} \mathrm{kpc}^{-2}$. Of the total FUV flux, $70 \%$ comes from the $\mathrm{H}$ i ring which is forming stars at a rate of $2.5 \times 10^{-3} M_{\odot} \mathrm{yr}^{-1}$. The gas consumption timescale, assuming a constant SFR and no gas recycling, is several times the age of the universe. In the context of the UV-optical galaxy color-magnitude diagram, the presence of the star-forming H i ring places NGC 404 in the green valley separating the red and blue sequences. The rejuvenated lenticular galaxy has experienced a merger-induced, disk-building excursion away from the red sequence toward bluer colors, where it may evolve quiescently or (if appropriately triggered) experience a burst capable of placing it on the blue/star-forming sequence for up to $\sim 1$ Gyr. The green valley galaxy population is heterogeneous, with most systems transitioning from blue to red but others evolving in the opposite sense due to acquisition of fresh gas through various channels.
\end{abstract}

Key words: galaxies: elliptical and lenticular, cD - galaxies: evolution - galaxies: individual (NGC 404) galaxies: interactions - galaxies: structure

\section{INTRODUCTION}

Strateva et al. (2001) used an early Sloan Digital Sky Survey (SDSS) galaxy catalog to demonstrate that the distribution of galaxies in color space is bimodal and correlated with galaxy morphology. Galaxies populate separate red and blue sequences in a $(u-r)$ versus $M_{r}$ color-magnitude diagram (CMD; Baldry et al. 2004). Evolution of galaxies from the blue sequence locus of actively star-forming systems to the red sequence occurs via transition through an intermediate CMD zone, christened the green valley (Martin et al. 2007, hereafter M07). Bell et al. (2004) and Faber et al. (2007) both have shown that the red sequence has grown in mass over the period from $z \sim 1$ to $z \sim 0$. Given that the color bimodality is driven principally by star formation activity and UV bands are an excellent tracer of recent star formation, it is not surprising that the galaxy color sequences are especially well separated in the UV-optical CMD (see Wyder et al. 2007, hereafter W07). Galaxy Evolution Explorer (GALEX) proved instrumental in characterizing the incidence of green valley transition galaxies (W07; M07) and understanding their evolution (M07; Schiminovich et al. 2007) from star-forming systems (Salim et al. 2007) to "red-and-dead" galaxies.

The effect of gas removal, and the subsequent quenching of star formation (SF), has been modeled (M07) in the context of galaxies evolving from the blue sequence to the red sequence across the green valley. However, the influence of late addition of gas to red sequence galaxies has received little attention. Contrary to classical expectations, galaxies of the types predominately populating the red sequence (elliptical and lenticular, S0) sometimes are associated with gaseous reservoirs, especially if they are in a low-density environment rather than within a cluster (Morganti et al. 2008). Oosterloo et al. (2007) showed that the structure of such gas is varied with some regularly rotating disks and a complement of extended, offset, even tail-like morphologies-suggesting a diversity in origin. Both galaxy mergers and intergalactic medium (IGM) accretion are viable mechanisms. The presence of centralized star formation in these early-type galaxies (ETGs) populating the red sequence has been associated with low angular momentum sources (such as retrograde mergers) because gas can be efficiently transported to the remnant center and consumed (Serra et al. 2008), leaving behind minimal H. On the other hand, prograde mergers of a red sequence galaxy with a gas-rich object may be the dominant mechanism of forming massive, extended $\mathrm{H}$ I distributions around ETGs.

NGC 404 is a prime target for studies addressing the structure and evolution of field S0 galaxies. It is particularly interesting because it is the nearest lenticular galaxy, lying just outside the Local Group at an estimated distance of $3.1-3.3 \mathrm{Mpc}$ 
(Karachentsev et al. 2002; Tonry et al. 2001). NGC 404 is presently isolated in a zone of radius $\sim 1.1 \mathrm{Mpc}$ (Karachentsev \& Makarov 1999). Although the galaxy is not affected by a cluster environment (as is true for many S0s), it may still have a complex history. Karachentsev \& Makarov (1999) suggested NGC 404 represents the end product in the coalescence of a small galaxy group, having accreted with all other (less massive) group members. At least one merger may have occurred in the recent past $(<1 \mathrm{Gyr})$ and be fundamentally responsible for the current UV morphology. del Río et al. (2004) argue that a large, disk-like, neutral atomic hydrogen $(\mathrm{HI})$ ring surrounding NGC 404 is the remnant of a merger with a dwarf irregular galaxy which took place some $900 \mathrm{Myr}$ ago, according to their kinematic estimation. Our GALEX observations revealed that this $\mathrm{H}_{\mathrm{I}}$ ring is now forming stars. This surprising, rather late discovery is understandable post facto since NGC 404 is nearly hidden in the glare of red supergiant Beta Andromedae (Mirach), $406^{\prime \prime}$ to the southeast of the galaxy. This bright foreground star makes it difficult to image the faint surface brightness portions of NGC 404 at red wavelengths (including $\mathrm{H} \alpha$ ), and the S0 galaxy became colloquially known as "Mirach's ghost."

In this Letter, we present evidence for star formation in NGC 404's outer disk-like H I ring. We refrain from detailed interpretation of UV properties of the inner disk and bulge. Section 2 describes our GALEX observations. Section 3 contains our data analysis. We conclude with discussion in Section 4.

\section{GALEX OBSERVATIONS}

We imaged NGC 404 with GALEX for 13463 s distributed over 11 visits during Fall 2008 in both the far- and nearultraviolet (FUV, NUV) bands as part of the Nearby Galaxies Survey (NGS). Our observations were co-added by the GALEX pipeline. The resulting deep FUV(NUV) image has $4^{\prime \prime} .2\left(5^{\prime \prime} .3\right)$ resolution (Morrissey et al. 2007).

The FUV band of GALEX effectively suppresses glare from Mirach and thus provides an unprecedented look at the starforming stellar population of NGC 404. However, UV imaging can be susceptible to extinction, effectively censoring the most embedded star formation events (Calzetti et al. 2005; Thilker et al. 2007b). In the outskirts of NGC 404, the expected extinction is thought to be low overall, as we describe in Section 3.1.

Figure 1 (top) presents a side-by-side comparison of red POSS-II, GALEX FUV, and H I (WHISP; van der Hulst et al. 2001) imaging for a wide field centered on NGC 404, encompassing the galaxy's entire low H I column density disk (detected until a radial distance of $\sim 800^{\prime \prime}, 12.8 \mathrm{kpc}$ ). The red light image gives no indication regarding the presence of the H I disk. However, we find that the main $\mathrm{H}$ I ring (between radii of $100^{\prime \prime}-400^{\prime \prime}$, 1.6-6.4 kpc) contains many FUV-bright sources, far in excess of the number expected from the background galaxy population. These GALEX sources are frequently unresolved or only marginally resolved, but often delineate larger aggregate structures (sizes of $\sim 0.1 \mathrm{kpc}$ to at least $2 \mathrm{kpc}$ ). The large-scale UV structures are correlated with the most prominent areas of $\mathrm{HI}$, though sometimes with a positional offset between associated features. The UV/H I morphology is suggestive of spiral waves triggering the star formation. We also note that at least in the $\mathrm{UV}$, the most significant H I peaks do not show as prominent SF sites. Some of the high $N(\mathrm{H}$ I) regions may be on the verge of forming the next generation of stellar clusters, but have not yet. We have plans to check for molecular gas concentrations matching the $N(\mathrm{HI})$ peaks.

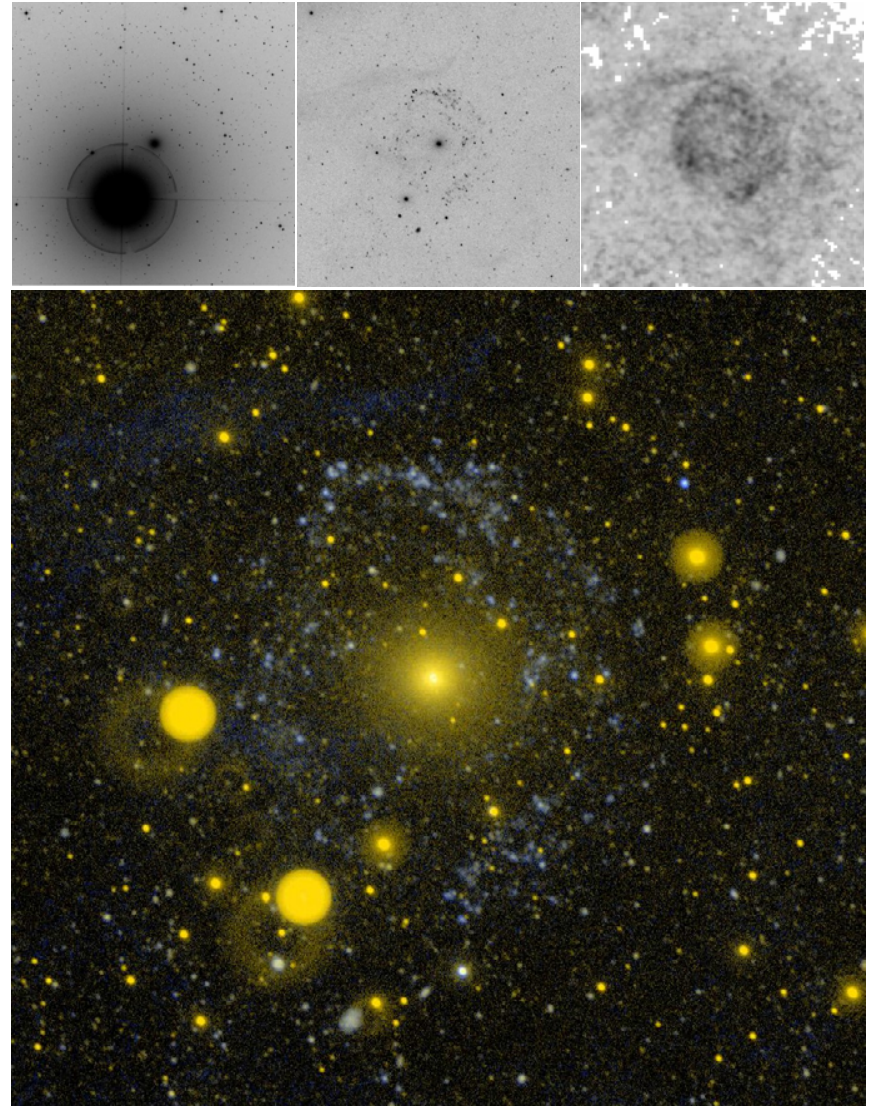

Figure 1. DSS2-red (top left), GALEX FUV (top center), H I (top right) imaging of a wide field centered on NGC 404, plus a detailed FUV, NUV color-composite image of NGC 404 (bottom). The FUV ( $\left.\lambda_{\text {eff }}=1538.6 \AA\right)$ imaging reveals star formation in the ring. The wide-field panels (at top) span $0.5 \times 0.5(27.3 \mathrm{kpc})$ with north up and east left. The GALEX color composite was created by assigning FUV to the blue channel, NUV ( $\lambda_{\text {eff }}=2315.7 \AA$ ) to the red channel, and an energy-weighted average of FUV and NUV to the green channel. FUVbright sources appear blue/white, including the majority of the detections in the SF ring. The inner disk and bulge appear yellow because it is NUV bright, dominated by older stars than the SF ring. Dichroic reflections appear as yellow doughnuts of varied sizes near bright foreground stars (including Mirach, SE of the galaxy). The color-composite field is $22^{\prime} .85 \times 21^{\prime} .80(20.8 \times 19.8 \mathrm{kpc})$.

We display a color-composite GALEX image of NGC 404 in the bottom panel of Figure 1. The inner disk and bulge of NGC 404 appear prominently in the NUV band, with average $\mathrm{FUV}-\mathrm{NUV}=1.8 \mathrm{AB}$ mag within $R_{25}\left(102^{\prime \prime}\right)$, as is typical for an S0 galaxy (Donas et al. 2007). The inner disk shows minimal substructure at $5^{\prime \prime}(80 \mathrm{pc})$ GALEX resolution, though other studies have described dust clouds (Tikhonov et al. 2003), molecular gas (Sage 1990; Wiklind \& Henkel 1990), and an ionized disk (Plana et al. 1998) within 200 pc of the LINER nucleus. The single most luminous FUV source in the galaxy occupies the nuclear area and is known to contain one dominant and several faint clusters of massive stars (Maoz et al. 1995, 1998). The most remarkable aspect of the color image in Figure 1 is the distribution of blue (FUV-bright) sources occupying the main SF H r ring. Typical FUV luminosity of individual clumps in this ring is $1.5 \times 10^{38} \mathrm{erg} \mathrm{s}^{-1}\left(m_{\mathrm{FUV}}=23.0 \mathrm{AB}\right.$ mag, or $M_{\mathrm{FUV}}=-5.6$ at $3.3 \mathrm{Mpc}$ ). All UV-related measurables in this Letter have been corrected only for Milky Way extinction, with $E(B-V)=0.059 \mathrm{mag}$ (Schlegel et al. 1998). Comparing intrinsic UV color to instantaneous burst Starburst99 models (Leitherer et al. 1999), we estimate that most sources have age $30 \mathrm{Myr}-1 \mathrm{Gyr}$ and corresponding stellar mass $10^{3}-10^{4} \mathrm{M}_{\odot}$. There is a sharp decline in the surface density of GALEX 


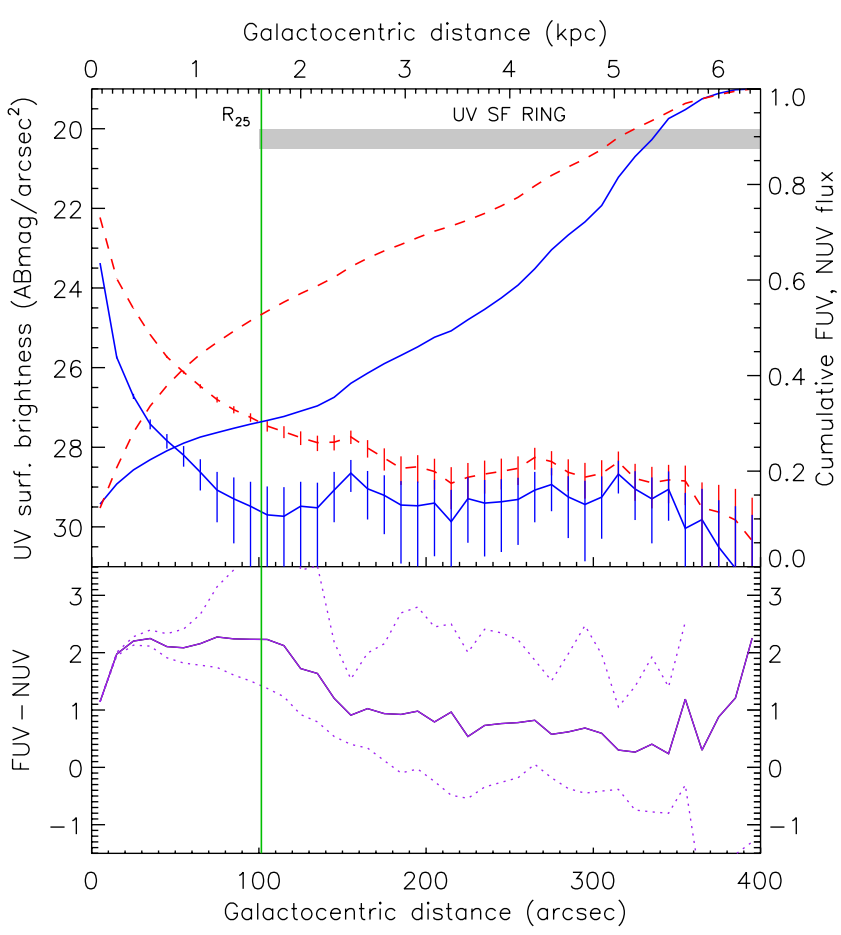

Figure 2. GALEX radial profile analysis for NGC 404. Top: average surface brightness and corresponding curve of growth as a function of galactocentric distance for the FUV (blue solid) and NUV (red dashed) GALEX bands. The $\pm 1 \sigma$ confidence intervals for the surface brightness profiles, including the uncertainty from sky subtraction, are plotted with thin color-coded lines. Bottom: azimuthally averaged FUV-NUV color with error limits (dotted lines), which are dominated by sky level uncertainty.

sources having blue FUV - NUV beyond a radius of $400^{\prime \prime}$. The $N(\mathrm{H}$ I) radial profile of del Río et al. (2004) indicates the average column density in this zone beyond the main ring is $\sim 4 \times 10^{19} \mathrm{~cm}^{-2}$. Two areas of exceptionally blue, yet diffuse FUV are seen-NE of the SF ring and overlapping with the ring to the E-SE. Their position within a larger filamentary network covering the GALEX field of view suggests they are attributable to Galactic cirrus.

\section{ANALYSIS AND RESULTS}

\subsection{Global Structure}

The top panel of Figure 2 shows background-subtracted, foreground-extinction corrected UV surface brightness profiles and growth curves measured for NGC 404 after masking of foreground stars and distant, unrelated galaxies. The bottom panel of Figure 2 presents FUV - NUV as a function of galactocentric distance. Table 1 lists integrated magnitudes and several quantities derived after folding in corollary data.

We neglect any average internal extinction, supported by inspection of Spitzer $24 \mu \mathrm{m}$ imaging of the SF ring obtained as part of the Local Volume Legacy Survey (Lee et al. 2009; Dale et al. 2009). Very few of the FUV-bright sources in NGC 404's ring have candidate $24 \mu \mathrm{m}$ counterparts. Indeed, the association of UV and IR emission in these cases may result from confusion of dusty background galaxies with UV ring clumps. Our extinction assumption is also supported by Prescott et al. (2007) who show that the fraction of highly obscured SF regions in ordinary galaxy disks is very small (4\%). Because we focus on a comparatively low-density, outer-disk environment, this is likely even more true in the NGC 404 ring (with at most a few significantly obscured SF complexes).
Table 1

Properties of NGC 404

\begin{tabular}{|c|c|c|c|}
\hline Quantity & Value & Unit & Reference \\
\hline Hubble type & $\mathrm{SA}(\mathrm{s}) 0$ & & (1) \\
\hline Morph. code, $t$ & $-2.8 \pm 0.6$ & & (2) \\
\hline Spectral (AGN) type & LINER & & (1) \\
\hline R.A. (J2000) & $01^{\mathrm{h}} 09^{\mathrm{m}} 27^{\mathrm{s}} .010$ & & (1) \\
\hline Decl. (J2000) & $+35^{\mathrm{d}} 43^{\mathrm{m}} 04^{\mathrm{s}} .20$ & & (1) \\
\hline Heliocentric velocity & $-48 \pm 9$ & $\mathrm{~km} \mathrm{~s}^{-1}$ & (1) \\
\hline Distance $^{\mathrm{a}}$ & 3.3 & $\mathrm{Mpc}$ & (3) \\
\hline$D_{25}$ & $203 \pm 14$ & $\operatorname{arcsec}$ & (2) \\
\hline$D_{\mathrm{H}_{\mathrm{I}}}$ & $\sim 1600$ & $\operatorname{arcsec}$ & (3) \\
\hline Inclination, $i$ & $0-11$ & deg & $(2,3)$ \\
\hline$M_{\mathrm{tot}}$ & $3 \times 10^{10}$ & $M_{\odot}$ & (3) \\
\hline$M_{\text {stellar }}$ & $6.9 \times 10^{8}$ & $M_{\odot}$ & (4) \\
\hline$M_{\mathrm{HI}}$ & $1.5 \times 10^{8}$ & $M_{\odot}$ & (3) \\
\hline$M_{\mathrm{tot}} / L_{B}$ & 85 & $M_{\odot} / L_{\odot}$ & (3) \\
\hline$M_{\mathrm{H}_{\mathrm{I}}} / L_{B}$ & 0.42 & $M_{\odot} / L_{\odot}$ & (3) \\
\hline $\operatorname{DEF}\left(\mathrm{H}_{\mathrm{I}}\right), \mathrm{H}_{\mathrm{I}}$ deficiency $^{\mathrm{b}}$ & -0.54 & & (3) \\
\hline$M_{\mathrm{HI}} / M_{\text {stellar }}$ & 0.22 & & $(3,4)$ \\
\hline Galactic $E(B-V)$ & 0.059 & mag & (1) \\
\hline $\mathrm{FUV}^{\mathrm{c}}$ & 14.89 & $\mathrm{AB}$ mag & (4) \\
\hline NUV & 13.68 & $\mathrm{AB}$ mag & (4) \\
\hline$r$ & 9.51 & $\mathrm{AB}$ mag & $(5,6)$ \\
\hline $\operatorname{FUV}\left(R<100^{\prime \prime}\right)$ & 16.20 & $\mathrm{AB}$ mag & (4) \\
\hline $\operatorname{NUV}\left(R<100^{\prime \prime}\right)$ & 14.39 & $\mathrm{AB}$ mag & (4) \\
\hline$r\left(R<100^{\prime \prime}\right)$ & 9.74 & $\mathrm{AB}$ mag & $(5,6)$ \\
\hline$L(\mathrm{FUV})$ & $1.0 \times 10^{41}$ & $\operatorname{erg~s}^{-1}$ & (4) \\
\hline $\mathrm{SFR}_{\mathrm{tot}}$ & $3.6 \times 10^{-3}$ & $M_{\odot} \mathrm{yr}^{-1}$ & (4) \\
\hline SFR $_{\text {ring }}$ & $2.5 \times 10^{-3}$ & $M_{\odot} \mathrm{yr}^{-1}$ & (4) \\
\hline$\Sigma_{\text {SFR,ring }}$ & $2.2 \times 10^{-5}$ & $M_{\odot} \mathrm{yr}^{-1}$ & (4) \\
\hline Gas consumption time, $\tau$ & 59 & Gyr & (4) \\
\hline $\mathrm{SFE}_{\mathrm{H}}$ & $1.7 \times 10^{-11}$ & $\mathrm{yr}^{-1}$ & (4) \\
\hline
\end{tabular}

Notes.

${ }^{a}$ We adopted 3.3 Mpc for consistency with del Río et al. (2004).

${ }^{\mathrm{b}}$ Based on the Haynes \& Giovanelli (1984) definition.

${ }^{c}$ All quantities below have been corrected for foreground extinction using the Cardelli et al. (1989) extinction law.

References. (1) NED; (2) HyperLEDA; (3) del Río et al. 2004; (4) this Letter; (5) Sandage \& Visvanathan 1978; (6) Tikhonov et al. 2003.

Figure 2 shows that (1) the main $\mathrm{H}$ I ring, at galactocentric distance $R=100^{\prime \prime}-400^{\prime \prime}$, has an average $\mu_{\mathrm{FuV}} \sim 29 \mathrm{AB} \mathrm{mag} /$ square arcsec; (2) FUV - NUV appears to slowly decline with increasing radius in the ring, from about 2 to 0.2 , probably reflecting contamination from an underlying exponential disk at least for smaller radii; (3) UV color for the inner disk/bulge is nearly constant at FUV - NUV $=2.2$ except for the nuclear area where FUV surface brightness increases (perhaps due to the LINER, or stronger UV upturn in central regions of the bulge; e.g., Ohl et al. 1998), bringing FUV - NUV down to 1.1. From the curve of growth, we determine that $70 \%$ of the total FUV luminosity $\left(L(\mathrm{FUV})=1.0 \times 10^{41} \mathrm{erg} \mathrm{s}^{-1}\right.$, corresponding to $\mathrm{FUV}=14.9$ ) in NGC 404 comes from the SF H I ring defined in (1). The H I ring has a star formation rate (SFR) of 0.0025 $M_{\odot} \mathrm{yr}^{-1}$, adopting the SFR calibration of Salim et al. (2007, their Equation (8)) for a Chabrier initial mass function (IMF) over $0.1-100 M_{\odot}$.

\subsection{Hubble Space Telescope Detection of a Resolved Young, Stellar Population}

Very deep F606W (39000s) and F814W (75400s) WFPC2 imaging of NGC 404 was obtained as part of ANGST (Dalcanton et al. 2009) and serendipitously samples a subsection 
of the SF ring $\left(R \sim 160^{\prime \prime}, 2.6 \mathrm{kpc}\right)$. We obtained the ANGST public-release photometric catalog of resolved stars from the Hubble Legacy Archive (HLA). Co-added images in F606W and F814W bands were retrieved from the ST-ECF Hubble Space Telescope (HST) Archive. At the color of the main sequence, ANGST observations of NGC 404 are 100\% complete for sources brighter than $\mathrm{F} 814 \mathrm{~W}=25.5 \mathrm{mag}$ and $50 \% \mathrm{com}$ plete down to $\sim 26.7 \mathrm{mag}$. This depth is sufficient to recover all massive main-sequence stars.

The NGC 404 ANGST data set will be described in a dedicated paper (D. A. Thilker et al. 2010, in preparation), but we note the following after initial image inspection: (1) there is minimal surface density enhancement within UV clumps, consistent with a single star or few stars emitting significant UV (cf. Gil de Paz et al. 2007); (2) occasional evidence for diffuse, nebular $(\mathrm{H} \alpha)$ emission in the $\mathrm{F} 606 \mathrm{~W}$ band is seen; (3) the overall distribution of resolved stars declines in surface density as a function of galactocentric distance, indicating the continued presence of an older, evolved disk (noted by Tikhonov et al. 2003 for a different WFPC2 field) at large $R$ underlying the ring; and (4) a small minority of the UV clumps may be contaminating sources such as background galaxies.

The HST data confirm that the UV light detected by GALEX originates from a young stellar population. CMD analysis of the ANGST catalogs in comparison to theoretical isochrones (Marigo et al. 2008) shows that in the UV clumps we detect main-sequence stars ranging in mass from $\sim 5$ to $40 M_{\odot}$, postturn-off stars (including the blue loop), and a background of red giant branch (RGB)/asymptotic giant branch (AGB) stars. Post-turn-off stars in the 100-300 Myr age range are also found outside of the UV clumps, suggesting stars from dispersed clusters may be present in the field.

\subsection{Star Formation Law and Threshold Considerations}

del Río et al. (2004) showed that gas surface density in the NGC 404 ring is subcritical in terms of the azimuthally averaged $Q$ parameter. Our data indicate that SF occurs in this environment, having average $\Sigma_{\mathrm{H}}=1.2 M_{\odot} \mathrm{pc}^{-2}$ between $R=$ $100^{\prime \prime}$ and $400^{\prime \prime}$. This is not surprising given other low-density SF detected by GALEX (XUV disks: Thilker et al. 2007a; ordinary spirals: Boissier et al. 2007; low-surface-brightness galaxies: Wyder et al. 2009; ETGs: Donovan et al. 2009). Local conditions must still be conducive to massive star formation. Indeed, clumps in the $\mathrm{H}_{\mathrm{I}}$ ring do reach $\Sigma_{\mathrm{H}_{\mathrm{I}}} \sim 4 M_{\odot} \mathrm{pc}^{-2}$.

However, the average SFR surface density in NGC 404's ring $\left(\Sigma_{\mathrm{SFR}} \sim 2.2 \times 10^{-5} M_{\odot} \mathrm{yr}^{-1} \mathrm{kpc}^{-2}\right)$ is remarkably low and provides further constraint on the SF law at low density. Even assuming negligible molecular content, the NGC 404 ring falls 1 dex below the traditional Kennicutt $\left(1998 ; \log \Sigma_{\mathrm{SFR}}, \log \Sigma_{\text {gas }}\right)$ correlation. The implied gas consumption timescale is $\sim 4 \times t_{H}$ (Hubble time, $1 / H_{0}$ ), without gas recycling. Equivalently, we infer SF efficiency of $1.7 \times 10^{-11} \mathrm{yr}^{-1}(0.16 \%$ over $100 \mathrm{Myr}$, or $>20 \times$ less than typical in galactic disks). The NGC 404 data are roughly consistent with the theoretical SF law of Krumholz et al. (2009), including a downturn in SF efficiency at low density due to a suppressed molecular fraction. The remaining offset (although small) is consistent with not accounting for a small contribution of molecular gas in the cloud cores of the ring-it must be comparatively rare, but still present. This is also true for the Wyder et al. (2009) sample of low surface brightness (LSB) galaxies.
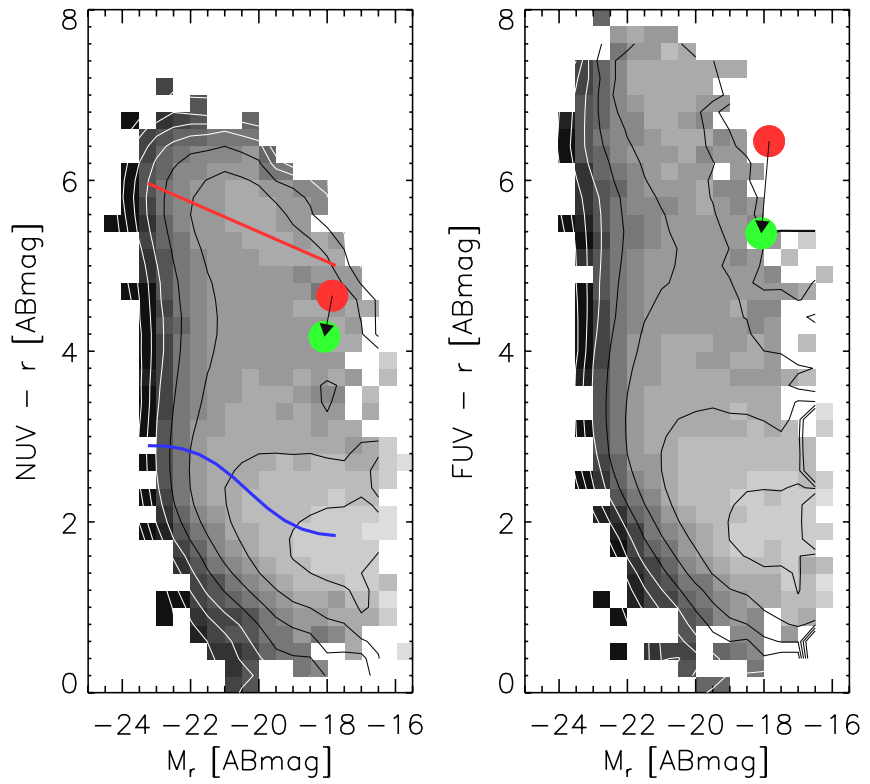

Figure 3. UV-optical galaxy CMDs (left, $\left(M_{r}, \mathrm{NUV}-r\right)$, right, $\left(M_{r}, \mathrm{FUV}-r\right)$ ) adapted from W07, upon which we have marked the position of NGC 404 both with and without the contribution of flux from the SF H I ring, using the green and red dots, respectively. In the $\left(M_{r}, \mathrm{NUV}-r\right) \mathrm{CMD}$ we overplot the W07 fits to the red and blue galaxy sequences. NGC 404 falls into the green valley when the ring is included. Galaxy volume density is gray-scaled over a range of 6 dex, with contours drawn every 0.5 dex.

\subsection{Backwards Evolution into the Green Valley}

In Figure 3, we plot the logarithm of volume density of galaxies in the UV-optical galaxy CMD. W07 fits to the red and blue $\left(\mathrm{M}_{r}, \mathrm{NUV}-r\right)$ sequences are marked. The green valley is the transitional region between these sequences. Conventionally, most green valley objects are thought to be evolving from the blue to the red sequence, after cessation of star formation activity. The GALEX photometry presented above is used to mark the position of NGC 404 in the galaxy CMD, both with (green) and without (red) the contribution of the $\mathrm{H}$ I ring. Optical magnitudes in both cases were determined by extrapolating the $r$-band, 84". 6 diameter aperture measurement of Sandage \& Visvanathan (1978) with the $I$-band surface brightness profiles of Tikhonov et al. (2003).

Assuming NGC 404's H I ring originates from a recent merger (del Río et al. 2004), then the galaxy has evolved from a red sequence object and moved backward into the green valley. We do not yet have a detailed recent star formation history to know if it is already returning to the red sequence or whether it will continue to evolve blueward. At the present SFR, it could indefinitely continue quiescently building $\left(\mathrm{SFR} / M_{*}=-11.4\right)$ a low-surface-brightness outer disk. Alternatively, if an epoch of enhanced SF were triggered perhaps by another encounter, consumption of the gas reservoir could position the galaxy on the blue/SF sequence $\left(\mathrm{SFR} / M_{*}=-9.4\right)$ for $\sim 1 \mathrm{Gyr}$. If the available $\mathrm{H}_{\mathrm{I}}$ in the ring were eventually all converted into stars, it could contribute an additional $\sim 20 \%$ to the stellar mass of NGC 404 (currently $6.9 \times 10^{8} M_{\odot}$ derived from spectral energy distribution (SED) fitting of UV-NIR integrated photometry), while substantially decreasing the bulge-to-disk mass ratio.

\section{DISCUSSION}

Star formation and associated gas are no longer viewed as uncommon in lenticular and elliptical galaxies, owing to 
ever more sensitive UV and $\mathrm{HI}$ imaging surveys. Residual star formation is thought to be present in $10 \%-30 \%$ of the ETGs examined with GALEX (Yi et al. 2005; Donas et al. 2007; Schawinski et al. 2007; Kaviraj et al. 2007), even after excluding classical UV-upturn candidate galaxies from the sample. An external source of gas (accretion or mergers) very likely supplements the SF fuel available via recycling from stellar mass loss. H I imaging for ETG samples supports this conclusion, frequently revealing extended gaseous distributions (Morganti et al. 2008). What remains to be determined is the long-term effect of externally fueled SF on the morphology of red-and-dead galaxies and on the appearance of the UV-optical galaxy CMD. Sometimes the gas is quickly consumed near the galaxy center (Serra et al. 2008), having little net effect on morphology and a very short-lived movement within the galaxy CMD. Activity of this sort is the "frosting" variety described by Trager et al. (2000), though Schawinski et al. (2009) also describe ETGs with rather high centralized SFRs ( $>50 M_{\odot} \mathrm{yr}^{-1}$ ). We have shown that rejuvenation of largescale disk formation is another possible outcome, as discovered in NGC 404 at a very low level. NGC 404 is classified as a Type 1 XUV disk using the criteria of Thilker et al. (2007a). Similar cases have been reported by Cortese \& Hughes (2009), Donovan et al. (2009), and Rich \& Salim (2009). Rejuvenation events may effectively transplant red sequence galaxies to the green valley and blue sequence. We note that $\mathrm{E} / \mathrm{S} 0$ galaxies have been detected already on the blue sequence (Kannappan et al. 2009; Schawinski et al. 2009). NGC 404 represents a possible example of this transition underway.

We conclude that the galaxy CMD transition zone known as the green valley represents a heterogeneous population of objects, with many evolving from blue to red but others going in the opposite direction. Stochastic excursions into the green valley from the red sequence, driven by acquisition of fresh gas for star formation, are observed. Traffic through the green valley is not one way. NGC 404 confirms that the merger of ETGs and gas-rich dwarfs are one mechanism establishing this diversity. This implies that UV imaging is fundamentally required to place individual ETGs into an accurate evolutionary context, assessing whether they are red and dead (at least for the moment), experiencing residual star formation in the galaxy center, or entering a rejuvenated phase of disk building fueled by a long-lasting gas reservoir. In fact, UV imaging is an effective means of detecting such potentially transformational reservoirs.

GALEX is a NASA Small Explorer, launched in 2003 April. We gratefully acknowledge NASA's support for construction, operation, and science analysis for the GALEX mission, developed in cooperation with the Centre National d'Etudes Spatiales of France and the Korean Ministry of Science and Technology. This research has made use of the NASA/IPAC Extragalactic Database (NED). We acknowledge the usage of the HyperLeda database (http://leda.univ-lyon1.fr). The Digitized Sky Surveys were produced at the Space Telescope Science Institute under U.S. Government grant NAG W-2166. Some images presented in this Letter were obtained from the Multimission Archive at the Space Telescope Science Institute (MAST).

\section{Facilities: GALEX, HST (WFPC2), VLA, WSRT}

\section{REFERENCES}

Baldry, I. K., Glazebrook, K., Brinkmann, J., Ivezić, Ž., Lupton, R. H., Nichol, R. C., \& Szalay, A. S. 2004, ApJ, 600, 681

Bell, E. F., et al. 2004, ApJ, 608, 752

Boissier, S., et al. 2007, ApJS, 173, 524

Calzetti, D., et al. 2005, ApJ, 633, 871

Cardelli, J. A., Clayton, G. C., \& Mathis, J. S. 1989, ApJ, 345, 245

Cortese, L., \& Hughes, T. M. 2009, MNRAS, 400, 1225

Dalcanton, J., et al. 2009, ApJS, 183, 67

Dale, D. A., et al. 2009, ApJ, 703, 517

del Río, M. S., Brinks, E., \& Cepa, J. 2004, AJ, 128, 89

Donas, J., et al. 2007, ApJS, 173, 597

Donovan, J. L., et al. 2009, AJ, 137, 5037

Faber, S. M., et al. 2007, ApJ, 665, 265

Gil de Paz, A., et al. 2007, ApJ, 661, 115

Haynes, M. P., \& Giovanelli, R. 1984, AJ, 89, 758

Kannappan, S. J., Guie, J. M., \& Baker, A. J. 2009, AJ, 138, 579

Karachentsev, I. D., \& Makarov, D. I. 1999, in IAU Symp. 186, Galaxy Interactions at Low and High Redshift, ed. J. E. Barnes \& D. B. Sanders (Berlin: Springer), 109

Karachentsev, I. D., et al. 2002, A\&A, 389, 812

Kaviraj, S., et al. 2007, ApJS, 173, 619

Kennicutt, R. C., Jr. 1998, ApJ, 498, 541

Krumholz, M. R., McKee, C. F., \& Tumlinson, J. 2009, ApJ, 699, 850

Lee, J. C., et al. 2009, ApJ, 706, 599

Leitherer, C., et al. 1999, ApJS, 123, 3

Maoz, D., Filippenko, A. V., Ho, L. C., Rix, H.-W., Bahcall, J. N., Schneider, D. P., \& Macchetto, F. D. 1995, ApJ, 440, 91

Maoz, D., Koratkar, A., Shields, J. C., Ho, L. C., Filippenko, A. V., \& Sternberg, A. 1998, AJ, 116, 55

Marigo, P., Girardi, L., Bressan, A., Groenewegen, M. A. T., Silva, L., \& Granato, G. L. 2008, A\&A, 482, 883

Martin, D. C., et al. 2007, ApJS, 173, 342 (M07)

Morganti, R., et al. 2008, in AIP Conf. Proc. 1035, The Evolution of Galaxies Through the Neutral Hydrogen Window, ed. R. Minchin \& E. Momjian (Melville, NY: AIP), 129

Morrissey, P., et al. 2007, ApJS, 173, 682

Ohl, R. G., et al. 1998, ApJ, 505, L11

Oosterloo, T. A., Morganti, R., Sadler, E. M., van der Hulst, T., \& Serra, P. 2007, A\&A, 465, 787

Plana, H., Boulesteix, J., Amram, P., Carignan, C., \& Mendes de Oliveira, C. 1998, A\&AS, 128, 75

Prescott, M. K. M., et al. 2007, ApJ, 668, 182

Rich, R. M., \& Salim, S. 2009, BAAS, 41, 252

Sage, L. J. 1990, A\&A, 239, 125

Salim, S., et al. 2007, ApJS, 173, 267

Sandage, A., \& Visvanathan, N. 1978, ApJ, 225, 742

Schawinski, K., et al. 2007, ApJS, 173, 512

Schawinski, K., et al. 2009, MNRAS, 396, 818

Schiminovich, D., et al. 2007, ApJS, 173, 315

Schlegel, D. J., Finkbeiner, D. P., \& Davis, M. 1998, ApJ, 500, 525

Serra, P., Trager, S. C., Oosterloo, T. A., \& Morganti, R. 2008, A\&A, 483, 57

Strateva, I., et al. 2001, AJ, 122, 1861

Thilker, D. A., et al. 2007a, ApJS, 173, 538

Thilker, D. A., et al. 2007b, ApJS, 173, 572

Tikhonov, N. A., Galazutdinova, O. A., \& Aparicio, A. 2003, A\&A, 401, 863

Tonry, J. L., Dressler, A., Blakeslee, J. P., Ajhar, E. A., Fletcher, A. B., Luppino, G. A., Metzger, M. R., \& Moore, C. B. 2001, ApJ, 546, 681

Trager, S. C., Faber, S. M., Worthey, G., \& González, J. J. 2000, AJ, 120, 165 van der Hulst, J. M., van Albada, T. S., \& Sancisi, R. 2001, in ASP Conf. Proc. 240, Gas and Galaxy Evolution, ed. J. E. Hibbard, M. Rupen, \& J. H. van Gorkom (San Francisco, CA: ASP), 451

Wiklind, T., \& Henkel, C. 1990, A\&A, 227, 394

Wyder, T. K., et al. 2007, ApJS, 173, 293 (W07)

Wyder, T. K., et al. 2009, ApJ, 696, 1834

Yi, S. K., et al. 2005, ApJ, 619, L111 